\title{
EVERY SUPERATOMIC SUBALGEBRA OF AN INTERVAL ALGEBRA IS EMBEDDABLE IN AN ORDINAL ALGEBRA
}

\author{
URI ABRAHAM AND ROBERT BONNET
}

(Communicated by Andreas R. Blass)

\begin{abstract}
Let us recall that a Boolean algebra is superatomic if every subalgebra is atnmic. So by the definition, every subalgebra of a superatomic algebra is superatomic. An obvious example of a superatomic algebra is the interval algebra generated by a well-ordered chain. In this work, we show that every superatomic subalgebra of an interval algebra is embeddable in an ordinal algebra, that is by definition, an interval algebra generated by a well-ordered chain. As a corollary, if $B$ is an infinite superatomic subalgebra of an interval algebra, then $B$ and the set $\operatorname{At}(B)$ of atoms of $B$ have the same cardinality.
\end{abstract}

\section{SURVEY OF THE RESULTS}

In a Boolean algebra $B$ we denote by $0_{B}$ and $1_{B}$, respectively the smallest and the largest elements of $B$. For $x$ and $y$ in $B$, we denote by $x \cup y$ and $x \cap y$, respectively, the supremum and the infimum of $x$ and $y$ in $B$, by $-x$ the complement of $x$ in $B$, and $x-y=x \cap(-y)$. So $-x=1_{B}-x-x$. Moreover, $x \subset y$ means $x \subseteq y$ and $x \neq y$.

For $0_{B} \neq a \in B$, we denote by $B \mid a$ the Boolean algebra induced by $B$ on the set $\{t \in B \mid t \subseteq a\}$. So $1_{B \mid a}=a$ and the complement of $t$ in $B \mid a$ is $a-t$.

For a Boolean algebra $B$ and a subset $D$ of $B$, we denote by $\operatorname{At}(B)$ the set of atoms $B$, and by $\operatorname{cl}_{B}(D)$ the subalgebra of $B$ generated by $D$.

For example, if $I$ is an ideal of $B$, then $\operatorname{cl}_{B}(I)=I \cup-I$, where $-I=\{-x \in$ $B \mid x \in I\}$.

Definition. A Boolean algebra $B$ is said to be superatomic if every quotient of $B$ is atomic.

Received by the editors December 14, 1989 and, in revised form, October 22, 1990.

1980 Mathematics Subject Classification (1985 Revision). Primary 06E05, 06E20, 54G12; Secondary $03 \mathrm{E} 05$.

Key words and phrases. Boolean algebras, interval algebras, superatomic Boolean algebras.

This work was a part of a lecture given by the second author, entitled "On Superatomic Boolean Algebras" to the NATO ASI meeting "Finite and Infinite Combinatorics and Logic" (Banff, Canada, April 21-May 4, 1991).

The first author was supported by the Université Claude-Bernard and the Ministère Français de l'Education Nationale.

The second author was supported by the Ministère Français des Affaires Etrangères, the C.N.R.S. (U.R.A. 225), and the Ben Gurion University of Negev. 
Day [3] (see also Koppelberg [5] and Roitman [7]) has shown the following result:

Proposition. Let $B$ be a Boolean algebra. The following properties are equivalent:

(i) B is superatomic;

(ii) every subalgebra of $B$ is atomic; and

(iii) there is no embedding from the atomless countable algebra into $B$.

If $E$ is a set, then $\wp(E)$, the power set of $E$, is regarded as a Boolean algebra.

Let $(C, \leq)$ be a partial ordered set. We say that $(C, \leq)$ is a chain if every pair of members of $C$ are comparable, and $(C, \leq)$ is well ordered if $(C, \leq)$ has no strictly decreasing sequence. Let $(C, \leq)$ be a chain with a first element denoted by $0_{C}$ (if $(C, \leq)$ has no first element, then we must add one). Let $C^{+} \stackrel{\text { def }}{=} C \cup\left\{\infty_{C}\right\}$ be the chain, obtained by adding a greatest element $\infty_{C}$. We denote by $B(C)$ the subalgebra of $\wp(C)$ generated by the set of $[a, b)$ for $a \in C$ and $b \in C^{+}$, i.e. $\operatorname{cl}_{\wp(C)}\left(\left\{[a, b) \mid a \in C\right.\right.$ and $\left.\left.b \in C^{+}\right\}\right) . B(C)$ is called the interval algebra on $C$ (see Koppelberg [5]).

Theorem 1. Let $B$ be a superatomic Boolean algebra. If $B$ is embeddable in an interval algebra, then $B$ is embeddable in an interval algebra generated by $a$ well-ordered chain $C$. More precisely, $B$ is isomorphic to a subalgebra $B^{\prime}$ of $B(C)$ and $\operatorname{At}\left(B^{\prime}\right)=\operatorname{At}(B(C))$.

Obviously Theorem 1 holds if $B$ is countable, since $B$ is isomorphic to an interval algebra (see Mayer and Pierce [6], Koppelberg [5]).

Example and comment. Let $B$ be a superatomic subalgebra of an interval algebra $B(C)$. The question is as follows: Is there a superatomic interval algebra $A$ such that $B \subseteq A \subseteq B(C)$ ? The answer is negative. Indeed, let $C=2 \cdot \lambda$, where $\lambda$ denotes the chain of real numbers, and thus $C$ is the chain obtained from the chain $\lambda$ by replacing each real number by the 2-elements chain. Let $B \stackrel{\text { def }}{=} \operatorname{cl}_{B(C)}(\operatorname{At}(B(C)))$. Let $A \stackrel{\text { def }}{=} B(D)$ be a superatomic interval algebra, generated by a chain $D$, containing $B$. First, because $\operatorname{At}(B)$ is uncountable, the scattered chain $D$ is uncountable. By a theorem of Hausdorff (see Rosenstein [8, Theorem 5.28]), $D$ contains a copy of the chain $\omega_{1}$ or $\omega_{1}^{*}$. For a contradiction, let us suppose that $B(D) \subseteq B(2 \cdot \lambda)$. Because $B(D)$ satisfies: there is an uncountable family of pairwise disjoint elements such that each element contains infinitely many atoms, the algebra $B(2 \cdot \lambda)$ has the same property. Now, we obtain a contradiction with the fact that in $\lambda$, every family of pairwise disjoint nontrivial intervals is at most countable.

For an infinite well-ordered chain $C$, the sets $\operatorname{At}(B(C))$ and $B(C)$ have the same cardinality; hence Theorem 1 implies the following result, proved by $M$. Rubin and S. Shelah [1988]:

Theorem 2. Let $B$ be an infinite superatomic Boolean algebra, embeddable in an interval algebra. Then $\operatorname{At}(B)$ and $B$ have the same cardinality.

This result completes the different characterizations of superatomic subalgebras of an interval algebra, developed by R. Bonnet, M. Rubin, and H. SiKaddour [1988]. Let us give an application of the above theorem. A Boolean 
algebra $B$ is said to be the thin-tall if $B$ is uncountable, and for each ordinal $\alpha$, the set $\operatorname{At}\left(D_{\alpha}(B)\right)$ is countable (the algebra $D_{\alpha}(B)$ is defined in 2.3). In particular $\operatorname{At}(B)$ is countable. From Theorem 2, for example, it follows that a thin-tall Boolean algebra is not embeddable in an interval algebra (for an application, see R. Bonnet and S. Shelah [2]).

\section{NotATION AND DEFINITION}

2.1. Chains. Let $(C, \leq)$ be a chain, $u \in C$, and $D$ be a subset of $C$.

$(C, \leq)$ is a complete chain if every subset of $C$ has a supremum and an infimum.

$(C, \leq)$ is a relatively complete chain if every bounded nonempty subset $A$ of $C$ (i.e. there are $b$ and $c$ in $C$ such that $b \leq a \leq c$ for every $a \in A$ ) has a supremum and an infimum in $C$.

Assume that $C$ is complete. We denote by $c(D, C)$, or more simply $c(D)$, the closure of $D$ in $C$ by supremum and infimum. For the following result, see e.g. Rosenstein [8]:

Every chain $(C, \leq)$ is embeddable in a complete chain $\left(C^{d}, \leq\right)$; namely, its Dedekind completion, (completion by cuts), which satisfies: for every $c \in C^{d}$, if $c$ is not the first element of $C^{d}$, then $c=\sup \{p \in C \mid p \leq c\}$, and if $c$ is not the last element of $C^{d}$, then $c=\inf \{p \in C \mid p \geq c\}$.

$u$ is a predecessor in $C$ if there is an (unique) element $u^{+} \in C$ such that $u^{+}>u$ and $\left[u, u^{+}\right)=\{u\}$. We denote by $\operatorname{Pred}(C)$ the set of predecessors of $C$.

$(C, \leq)$ is totally disconnected if for every $v<w$ in $C$, we have $[v, w) \cap$ $\operatorname{Pred}(C) \neq \varnothing$. Consequently $B(C)$ is atomic if and only if $C$ is totally disconnected. The word "totally disconnected" comes from the fact that a chain $C$ is totally disconnected if and only if $C$, endowed with the interval topology, is a totally disconnected space.

Let $\cong$ be an equivalence relation on $C$. For $a \in C$, we denote by $a / \cong$ its equivalence class, i.e. $a / \cong$ def $=\left\{a^{\prime} \in C \mid a^{\prime} \cong a\right\}$. We suppose that each equivalence class is an interval of $C$. For $\tilde{a}^{\prime}, \tilde{a}^{\prime \prime} \in C / \cong$ we set $\tilde{a}^{\prime}<\tilde{a}^{\prime \prime}$ if for every $a^{\prime} \in \tilde{a}^{\prime}$ and $a^{\prime \prime} \in \tilde{a}^{\prime \prime}$, we have $a^{\prime}<a^{\prime \prime}$. If each equivalence class is an interval of $C$, then $(C / \cong, \leq)$ is a chain. Moreover, if $(C, \leq)$ is a complete chain, then $(C / \cong, \leq)$ is too.

2.2. Boolean algebras. An element $a$ of an interval algebra $B(C)$, different from $0_{B}$, has a unique decomposition (called the canonical decomposition), of the form: $a=\bigcup\left\{\left[a_{2 i}, a_{2 i+1}\right) \mid i<n\right\}$ where $0<n<\omega, 0_{C} \leq a_{0}<a_{1}<a_{2}<$ $\cdots<a_{2 n-1} \leq \infty_{C}$, and $a_{k} \in C^{+} \stackrel{\text { def }}{=} C \cup\left\{\infty_{C}\right\},(k=0,1, \ldots, 2 n-1)$. For such an element $a$, we set $\sigma(a)=\left\{a_{k} \mid k<2 n\right\} \subseteq C \cup\left\{\infty_{C}\right\}$. The integer $n$ is called the length of $a$, and is denoted by $l(a)$.

Every finite product of interval algebras is isomorphic to an interval algebra, and thus if $B$ is a subalgebra of a finite product of interval algebras, then $B$ is embeddable in an interval algebra.

More precisely, let us recall the following fact concerning chains and Boolean algebras (see [5, Proposition 15.11]). Let $C_{1}$ and $C_{2}$ be chains with first element $0_{C_{1}}$ and $0_{C_{2}}$ respectively. Let $C=C_{1}+C_{2}$ be the chain, lexicographic sum of $C_{1}$ and $C_{2}$ (so $c_{1}<c_{2}$ for $c_{1} \in C_{1}$ and $c_{2} \in C_{2}$ ). Note that $C$ has 
a first element, namely $0_{C_{1}}$. A canonical isomorphism $f$ from $B(C)$ onto $B\left(C_{1}\right) \times B\left(C_{2}\right)$ is obtained by letting: $f(c)=\left\langle c \cap C_{1}, c \cap C_{2}\right\rangle$. Let us remark that we identified $\infty_{C_{1}}$ with $0_{C_{2}} . B\left(C_{1}\right), B\left(C_{2}\right)$ are factors of $B(C)$; and by identification, $B(C)\left\lceil C_{1}=B\left(C_{1}\right)\right.$ and $B(C)\left\lceil C_{2}=B\left(C_{2}\right)\right.$.

Let $D$ be a subset of $C$, containing $0_{C}$. Hence $D$ is a chain with a first element. We denote by $B_{C}(D)$ the subalgebra of $B(C)$ consisting of those elements $a$ such that $\sigma(a) \subseteq D \cup\left\{\infty_{C}\right\}$. Let us remark that the Boolean algebras $B_{C}(D)$ and $B(D)$ are isomorphic.

2.3. Let $B$ be a Boolean algebra. By induction, we define a sequence $\left(I_{\alpha}(B)\right.$, $\left.D_{\alpha}(B), \pi_{\alpha}^{B}\right)$, with the conditions $D_{\alpha}(B)=B / I_{\alpha}(B)$ and $\pi_{\alpha}^{B}$ is the canonical homomorphism from $B$ onto $D_{\alpha}(B)$ (the algebra $D_{\alpha}(B)$ is called the $\alpha$-th Cantor Bendixon derivative of $B$ ). Let $I_{0}(B)=\{0\}$, and thus $D_{0}(B)=B$. Suppose that $I_{\alpha}(B)$ has been defined. Let $J_{\alpha}(B)$ be the ideal of $D_{\alpha}(B)$, generated by $\operatorname{At}\left(D_{\alpha}(B)\right)$. Then $I_{\alpha+1}(B) \stackrel{\text { def }}{=}\left(\pi_{\alpha}^{B}\right)^{-1}\left(J_{\alpha}(B)\right)$. Suppose that $\delta$ is a limit, and $I_{\alpha}(B)$ has been defined for every $\alpha<\delta$, then $I_{\delta}(B)=\bigcup_{\alpha<\delta} I_{\alpha}(B)$.

The following additional equivalences are well known and their proofs are straightforward (see Koppelberg [5]).

Proposition. Let $B$ be a Boolean algebra. The following properties are equivalent:

(i) $B$ is superatomic, and

(ii) there is an ordinal $\gamma$ such that $1_{B} \in I_{\gamma}(B)$.

Clearly the first ordinal $\gamma$ for which $1_{B} \in I_{\gamma}(B)$ is a successor ordinal, say $\alpha+1$, and $\alpha$ is $\alpha$ is denoted by $\operatorname{rk}(B)$. Hence $1_{B} \in I_{\mathrm{rk}(B)+1}(B)-I_{\mathrm{rk}(B)}(B)$ and $D_{\mathrm{rk}(B)}(B)$ is a nontrivial finite algebra isomorphic to $\wp(n) \quad(n>0$ integer), and if $n=1$, then $I_{\mathrm{rk}(B)}(B)$ is a maximal ideal of $B$. Let $I(B)$ and $D(B)$ denote $I_{\mathrm{rk}(B)}(B)$ and $B / I(B)$ respectively.

For $b \in B, b \neq 0_{B}$ let $\operatorname{rk}_{B}(b)$ be the first ordinal $\alpha$ such that $b \notin I_{\alpha}(B)$. Hence $b \in I_{\mathrm{rk}_{B}(b)+1}(B)-I_{\mathrm{rk}_{B}(b)}(B)$. For instance $\operatorname{rk}_{B}(b)=0$ for $b \in \operatorname{At}(B)$, and $\operatorname{rk}_{B}\left(1_{B}\right)=\operatorname{rk}(B)$.

Notation 2.4. Let $B$ be a subalgebra of an algebra $A$, and $c \in A$. We denote by $B\lceil c$ the set of $b \cap c$ for $b \in B$. We regard $B\lceil c$ as a subalgebra of the factor $A\lceil c$ of $A$, and thus as a Boolean algebra. Remark that if $c \in B$, then $B\lceil C$ is a factor of $B$.

By the definition, $B \mid C$ is an homomorphic image of $B$. From the fact that, for every superatomic Boolean algebra $A$ and every ideal $I$ of $A$, we have $\operatorname{rk}(A / I) \leq \operatorname{rk}(A)$, it follows that:

Lemma 2.5. Let $B$ be a subalgebra of a superatomic Boolean algebra $A$, and $c \in A$. Then $\operatorname{rk}(B \mid c) \leq \operatorname{rk}(B)=\operatorname{rk}_{B}\left(1_{B}\right)$.

The following result is due to M. Rubin and S. Shelah [9], and is one of the ingredients of the original proof of Theorem 2.

Proposition 2.6 (Rubin and Shelah). Let $B$ be an atomic subalgebra of an interval algebra. Then there are a totally disconnected complete chain $C$ and an embedding $\phi$ from $B$ into $B(C)$ such that $B(C)$ is an atomic algebra and $\operatorname{At}(\phi[B])=\operatorname{At}(B(C))$. 
Note that the property of $C$ implies that $c(\bigcup \operatorname{At}(B(C)), C)=C$. The proof of Proposition 2.6 needs some preliminary results. Let $C$ be a chain such that $B \subseteq B(C)$.

Claim 2.7. We can suppose that $C$ satisfies (1): $C$ is a complete chain, and (2): every atom of $B$ is a finite subset of $C$.

Proof. We can suppose that $C$ is a complete chain (consider its Dedekind completion). Let $\underline{C}=\bigcup\{\sigma(a) \mid a \in B\}$ be the set of endpoints of elements of $B$. We set $\underline{C}^{c}=c(\underline{C}, C)$. The function $\phi$ from $B$ into the subalgebra $B_{\underline{C}^{c}}(\underline{C})$ of $B\left(\underline{C}^{c}\right)$ defined by $\phi(b)=b \cap \underline{C}$ is trivially a one-to-one homomorphism, and $\underline{C}^{c}$ is as required (note that by the construction, (2) is satisfied).

Claim 2.8. We can suppose that $C$ satisfies (1) and (3): every atom of $B$ is a singleton of $C$.

Proof. For every $a \in \operatorname{At}(B)$, we have $a=\bigcup_{i<l(a)}\left[a_{2 i}, a_{2 i+1}\right)=\bigcup_{i<l(a)}\left\{a_{2 i}\right\}$ with $a_{k} \in C^{+}=C \cup\left\{\infty_{C}\right\}$, and $a_{2 i}, a_{2 i+1}$ consecutive in $C$. Let $\sim$ be the equivalence on $C$ defined by $a_{2 i} \sim a_{2 i+1}$ for $0<i<l(a)$ and $a \in \operatorname{At}(B)$. Let $\underline{C}=C / \sim$. Hence $\underline{C}$, with the induced linear order by $C$, is a complete chain. Let $\varphi$ be the function from $B(C)$ into $B(\underline{C})$ defined as follows: if $b=\bigcup_{i<l(b)}\left[b_{2 i}, b_{2 i+1}\right)$, then $\varphi(b)=\bigcup_{i<l(b)}\left[b_{2 i} / \sim, b_{2 i+1} / \sim\right)$. Obviously $\varphi$ is a homomorphism from $B(C)$ onto $B(\underline{C})$. It suffices to show that $\varphi(a)=$ $\left[a_{0} / \sim, a_{1} / \sim\right)=\left\{a_{0} / \sim\right\} \neq 0$ for $a \in \operatorname{At}(B)$, and $\varphi$ restricted to $B$ is one-to-one. But this is trivial.

Proof of Proposition 2.6. To prove the proposition, there is no loss in assuming that $B$ and $C$ satisfy the assumptions (1) and (3) of Claim 2.8. Let $\equiv$ be the equivalence on $C$ defined by $x \equiv y$ if $x=y$, or if $x \leq y$ and $[x, y)$ does not contain an atom of $B$, or if $y \leq x$ and $[y, x)$ does not contain an atom of $B$. Then the quotient chain $C \stackrel{\text { def }}{=} C / \equiv$ is complete and totally disconnected. Let $\rho$ be the canonical increasing function from $C$ onto $\underline{C}$. Note that if $x<y$ in $C$ are such that $\rho(x)<\rho(y)$ in $\underline{C}$, then there is $a \in \operatorname{At}(B)$ such that $a \subseteq[x, y)$. This shows that the function $\phi$ from $B$ into $B(\underline{C})$ defined by $\phi(a)=\bigcup\left\{\left[\rho\left(a_{2 i}\right), \rho\left(a_{2 i+1}\right)\right) \mid i<l(a)\right\}$ for $a=\bigcup\left\{\left[a_{2 i}, a_{2 i+1}\right) \mid i<l(a)\right\}$ in $B \subseteq B(C)$, is as required, and satisfies $\operatorname{At}(\phi[B])=\operatorname{At}(B(\underline{C}))$. That finishes the proof of Proposition 2.6.

\section{Proof of Theorem 1}

3.1. To prove Theorem 1 , there is no loss in assuming that (1): $B$ satisfies both the premises and the conclusions of Proposition 2.6, and (2): $I(B)=I_{\mathrm{rk}(B)}(B)$ is a maximal ideal of $B$. We denote by $\cong$ the relation on $C$ defined by $x \cong y$ if $x \leq y$ and there is $b \in B$, with $\operatorname{rk}_{B}(b)<\mathrm{rk}_{B}\left(1_{B}\right)$, containing $[x, y)$, or $y \leq x$ and there is $b \in B$, with $\operatorname{rk}_{B}(b)<\operatorname{rk}_{B}\left(1_{B}\right)$, containing $[y, x)$. We show:

Lemma 3.2. Each equivalence class is an interval of $C$. Let $b \in B$ be such that $\mathrm{rk}_{B}(b)<\mathrm{rk}_{B}\left(1_{B}\right)$. Then there is a finite subset $a_{0} / \cong, a_{1} / \cong, \ldots, a_{n-1} / \cong$ of equivalence classes such that $b \subseteq \bigcup\left\{a_{k} / \cong \mid k<n\right\}$.

Proof. The first part of the claim is trivial. Let us show the second one. Let 
$b=\bigcup\left\{\left[b_{2 i}, b_{2 i+1}\right) \mid i<n\right\}$. It suffices to show that $b_{2 i} \cong b_{2 i+1}$ for $i<n$. But this is a trivial consequence of the definition of $\cong$.

The following two claims are obvious.

Claim 3.3. If $a \in \operatorname{At}(B)$, then $a$ is contained in an equivalence class.

Claim 3.4. Let $\tilde{a}$ be an equivalence class. If $\tilde{a}$ has a last element $v$, then $v \notin \operatorname{Pred}(C)$.

Definition 3.5. Let $C$ be a complete totally disconnected chain, $B$ a superatomic subalgebra of $B(C), \lambda$ an ordinal, and $\psi$ a function from $B$ into $B(\lambda)$. We say that $(B, C, \lambda, \psi)$ is a good system if $\operatorname{At}(B)=\operatorname{At}(B(C)), \psi$ is a one-to-one homomorphism from $B$ into the interval algebra $B(\lambda)$, and the restriction $\psi \mid \operatorname{At}(B)$ of $\psi$ on $\operatorname{At}(B)$ is a one-to-one function from $\operatorname{At}(B)$ onto $\operatorname{At}(B(\lambda))$. We say that there is a good system for $(B, C)$ if there are $\lambda$ and $\psi$ such that $(B, C, \lambda, \psi)$ is a good system.

Note that $\operatorname{At}(\psi[B])=\psi[\operatorname{At}(B)]$. Equivalently a good system is the $(B, C$, $\left.\lambda, \psi_{0}\right)$, where $\operatorname{At}(B)=\operatorname{At}(B(C))$, and $\psi_{0}$ is a one-to-one function from $\operatorname{Pred}(C)$ into the chain $\lambda$ such that the function $\underline{\psi}_{0}$ from $\operatorname{At}(B(C))$ into $\operatorname{At}(B(\lambda))$ defined by $\psi_{0}\left(\left[u, u^{+}\right)\right)=\left[\psi_{0}(u), \psi_{0}(u)+1\right)$ for $u \in \operatorname{Pred}(C)$ can be extended in an embedding $\psi$ from $B$ into $B(\lambda)$.

We prove by induction on $\alpha$, that the following statement $\operatorname{Th}(\alpha)$ holds: for every chain $C$ and for every superatomic subalgebra $B$ of $B(C)$, such that $\operatorname{rk}(B) \leq \alpha$ and $\operatorname{At}(B)=\operatorname{At}(B(C))$, there is a good system for $(B, C)$.

$\mathrm{Th}(0)$ and $\mathrm{Th}(1)$ hold. Indeed $B$ is isomorphic to the Boolean algebra $F_{c}(X)$ of finite or cofinite subsets of a set $X$, where $X=\operatorname{At}(B(C)$ ) (since $I(B)=I_{\mathrm{rk}(B)}(B)$ is a maximal ideal of $\left.B\right)$. Consider $\lambda$ be the (initial) ordinal corresponding to the cardinality of the set $\operatorname{At}(B(C))$. In what follows, we suppose that $\operatorname{rk}(B) \geq 2$.

Claim 3.6. Let $\tilde{a} /$ cong be an equivalence class, and

$$
\tilde{a} \stackrel{\text { def }}{=}((a / \cong) \cup\{\inf (a / \cong)\})-\{\max (a / \cong)\}=[\inf (a / \cong), \sup (a / \cong)) \text {. }
$$

There is a good system for for $(B \backslash \tilde{a}, \tilde{a})$.

Proof. By induction. If $\tilde{a}=\{a\}$, then it is trivial. Assume $|\tilde{a}| \geq 2$. Let $c \in \operatorname{Pred}(\tilde{a}), \tilde{a}^{+}=\{x \in \tilde{a} \mid x>c\}$, and $\tilde{a}^{-}=\{x \in \tilde{a} \mid x \leq c\}$. Note that $\tilde{a}$ is the lexicographic sum $\tilde{a}^{-}+\tilde{a}^{+}$, and $\tilde{a}^{+}$has a first element (the successor of $c$ ). If $\tilde{a}^{-}$has no first element, then we must add one, namely $\inf (a / \cong)$. Suppose that $\left(B \backslash \tilde{a}^{+}, \tilde{a}^{+}, \lambda^{+}, \psi^{+}\right)$and $\left(B \backslash \tilde{a}^{-}, \tilde{a}^{-}, \lambda^{-}, \psi^{-}\right)$are good. Let $\left(B \backslash \tilde{a}, \tilde{a}, \lambda^{-}+\lambda^{+}, \psi\right)$, where $\psi$ is defined in the following way: for $b \in B \backslash \tilde{a}$, we have $b=\left\langle b^{-}, b^{+}\right\rangle \in B\left(\tilde{a}^{-}\right) \times B\left(\tilde{a}^{+}\right)$and we set $\psi(b)=\left\langle\psi^{-}\left(b^{-}\right), \psi^{+}\left(b^{+}\right)\right\rangle \in$ $B\left(\lambda^{-}\right) \times B\left(\lambda^{+}\right)$(that is identified with $B\left(\lambda^{-}+\lambda^{+}\right)$). Trivially, $\left(B \mid \tilde{a}, \tilde{a}, \lambda^{-}+\right.$ $\left.\lambda^{+}, \psi\right)$ is as required. So, it suffices to prove that Claim 3.6, whenever $\tilde{a}^{+}=\tilde{a}$ or $\tilde{a}^{-}=\tilde{a}$. We prove the case $\tilde{a}^{+}=\tilde{a}$. The case $\tilde{a}^{-}=\tilde{a}$ is similar: but note that if $a / \cong$ has no first element, then $\tilde{a}=(a / \cong) \cup\{\inf (a / \cong)\}$, and the algebras

$$
B \backslash(a / \cong) \text { and } B\lceil((a / \cong) \cup\{\inf (a / \cong)\})
$$


are isomorphic. $\tilde{a}^{+}=\tilde{a}$ satisfies: $\tilde{a}$ has a first element, denoted by $e$, and for every element $x$ of $\tilde{a}$, we have $x \cong e$.

Case 1. $a / \cong$ has a last element $e^{+}$. Hence $e^{+} \cong e$ and $\tilde{a} \stackrel{\text { def }}{=}(a / \cong)$ $-\left\{e^{+}\right\}=[e, e+)$ (that is the case of the example which follows from Theorem $1)$. Let $b \in B$ be such that $\tilde{a} \subseteq b$, and $\operatorname{rk}_{B \nmid b}\left(1_{B \nmid b}\right)=\operatorname{rk}_{B}(b)<\operatorname{rk}_{B}\left(1_{B}\right)$. Let $B \nmid \tilde{a} \stackrel{\text { def }}{=}\{c \cap \tilde{a} \mid c \in B\}$. Note that $B \backslash \tilde{a}=(B \nmid b) \uparrow \tilde{a}$. We regard $B \backslash \tilde{a}$ as a Boolean algebra. By the definition, $B \backslash \tilde{a}$ is a homomorphic image of $B \backslash b$. From the fact that for every superatomic Boolean algebra $A$ and every ideal $I$ of $A$, we have $\operatorname{rk}(A / I) \leq \operatorname{rk}(A)$, it follows that $\operatorname{rk}(B \mid \tilde{a}) \leq \operatorname{rk}(B \backslash b)=$ $\operatorname{rk}_{B}(b)<\operatorname{rk}_{B}\left(1_{B}\right)=\operatorname{rk}(B)$. By the induction hypothesis there is a good system for $(B \backslash \tilde{a}, \tilde{a})$.

Case 2. $a / \cong$ has no last element. Hence $\tilde{a}=a / \cong$. Let $\left(e_{\alpha}\right)_{\alpha<\sigma}$ be a stricly increasing sequence, cofinal in $\tilde{a}$. We can suppose that $e_{0}=e$, and $e_{\beta}=\sup \left\{e_{\alpha} \mid \alpha<\beta\right\}$ for every limit ordinal $\beta<\sigma$ (because $\tilde{a}$ is relatively complete). Let $\alpha<\sigma$ be given. Let $b_{\alpha} \in B$ be such that $\operatorname{rk}_{B}\left(b_{\alpha}\right)<\operatorname{rk}_{B}\left(1_{B}\right)$ and $\left[e_{\alpha}, e_{\alpha+1}\right) \subseteq b_{\alpha}$. We set $B_{\alpha} \stackrel{\text { def }}{=} B \backslash\left[e_{\alpha}, e_{\alpha+1}\right)$. Since Lemma 2.5 , we have $\operatorname{rk}\left(B_{\alpha}\right) \leq \operatorname{rk}\left(B \mid b_{\alpha}\right)=\operatorname{rk}_{B}\left(b_{\alpha}\right)<\operatorname{rk}_{B}\left(1_{B}\right)=\operatorname{rk}(B)$. Applying the induction hypothesis to $\left(B_{\alpha},\left[e_{\alpha}, e_{\alpha+1}\right)\right)$, there is a good system $\left(B_{\alpha},\left[e_{\alpha}, e_{\alpha+1}\right), \mu_{\alpha}, \psi_{\alpha}\right)$. Hence $\psi_{\alpha}\left[\left[e_{\alpha}, e_{\alpha+1}\right)\right]=\mu_{\alpha}$. Let $\mu=\sum_{\alpha<\sigma} \mu_{\alpha}$, and $\psi=\bigcup\left\{\psi_{\alpha} \mid \alpha<\sigma\right\}$. We have $\operatorname{Pred}(\mu)=\mu$. We extend $\psi$ in an one-to-one homomorphism $\psi$ from $B \nmid \tilde{a}$ into $B(\mu)$ : let $b \in B$. We set:

$\underline{\psi}(b)=\bigcup\left\{\mu_{\alpha} \mid\left[e_{\alpha}, e_{\alpha+1}\right) \subseteq b\right\} \cup \bigcup\left\{\psi_{\alpha}\left(b \cap\left[e_{\alpha}, e_{\alpha+1}\right)\right) \mid b \cap\left[e_{\alpha}, e_{\alpha+1}\right) \neq 0_{B_{\alpha}}, 1_{B_{\alpha}}\right\}$.

We must remark that $\psi$ is well defined, because $b$ is a finite union of halfopen intervals and thus $\left\{\alpha<\sigma \mid\left[e_{\alpha}, e_{\alpha+1}\right) \subseteq b\right\} \in B(\sigma)$, and the set $\{\alpha<$ $\left.\sigma \mid b \cap\left[e_{\alpha}, e_{\alpha+1}\right) \neq 0_{B_{\alpha}}, 1_{B_{\alpha}}\right\}$ is finite. Consequently $\psi(b)$ is a finite union of half-open intervals of $\mu$, and thus $(B \backslash \tilde{a}, \tilde{a}, \mu, \underline{\psi})$ is a good system. That finishes the proof of Claim 3.6.

End of the proof of Theorem 1. Let $\left(a_{\zeta} / \cong\right)_{\zeta<\theta}$ be an enumeration of the set of equivalence classes. By Claim 3.6, for $\zeta<\theta$, let $\left(B \backslash \tilde{a}_{\zeta}, \tilde{a}_{\zeta}, \lambda_{\tilde{a}_{\zeta}}, \psi_{\tilde{a}_{\zeta}}\right)$ be a good system. Let $\lambda=\sum_{\zeta<\theta} \lambda_{\tilde{a}_{\zeta}}$. Each $\lambda_{\tilde{a}_{\zeta}}$ is an interval of $\lambda$. Now, let $\psi$ be the function from $\operatorname{At}(B)$ into $\lambda$ defined by $\psi(a)=\psi_{\tilde{a}_{\zeta}}(a)$ where $\tilde{a}_{\zeta}$ is the unique class such that $a \in \operatorname{At}(B) \cap B \mid \tilde{a}_{\zeta}$. Let $b \in B$. First, suppose that $\operatorname{rk}_{B}(b)<\operatorname{rk}_{B}\left(1_{B}\right)$. There is a finite subset $\left\{\tilde{a}_{0}, \tilde{a}_{1}, \ldots, \tilde{a}_{n-1}\right\}$ of equivalence classes such that $b \subseteq \bigcup\left\{\tilde{a}_{k} \mid k<n\right\}$, follows from Lemma 3.2. We set $\psi(b)=\bigcup\left\{\psi_{\tilde{a}_{k}}\left(b \cap \tilde{a}_{k}\right) \mid k<n\right\}$. Now, because $I(B)=I_{\mathrm{rk}(B)}(B)$ is a maximal ideal of $B$, if $\mathrm{rk}_{B}(b)=\mathrm{rk}_{B}\left(1_{B}\right)$, then $\mathrm{rk}_{B}(-b)<\mathrm{rk}_{B}\left(1_{B}\right)$, and we set $\psi(b)=-\psi(-b)$. The fact that $\psi$ is a one-to-one homomorphism from $B$ into $B(\lambda)$ is a consequence of the following obvious result:

Claim 3.7. Let $B^{\prime}$ and $B^{\prime \prime}$ be two atomic algebras and $\psi$ be a one-to-one function from $\operatorname{At}\left(B^{\prime}\right)$ onto $\operatorname{At}\left(B^{\prime \prime}\right)$. We suppose that, for each $b \in B^{\prime}$, there is an unique element of $B^{\prime \prime}$, denoted by $\psi(b)$, such that for every $a \in \operatorname{At}\left(B^{\prime}\right)$, we have $a \subseteq b$ if and only if $\psi(a) \subseteq \psi(b)$. Then $\psi$ is a one-to-one homomorphism from $B^{\prime}$ into $B^{\prime \prime}$, extending $\psi$. This finishes the proof of Theorem 1. 


\section{ACKNOWLEDGMENT}

We thank Matatyahu Rubin, Maurice Pouzet, and Hamza Si-Kaddour for comments during the preparation of this paper.

\section{REFERENCES}

1. R. Bonnet, M. Rubin, and H. Si-Kaddour, On Boolean algebras with well-founded set of generators, Trans. Amer. Math. Soc., submitted.

2. R. Bonnet and S. Shelah, On HCO spaces. An uncountable compact $T_{2}$ space, different of $\aleph_{1}+1$, which is homeomorphic to each of its uncountable closed subspace, Israel J. Math., (second version, 1991, submitted).

3. G. W. Day, Superatomic Boolean algebras, Pacific J. Math. 23 (1967), 479-489.

4. P. Halmos, Lectures on Boolean algebras, Van Nostrand Math. Studies, vol. 1, Van Nostrand Co., NY, 1963.

5. S. Koppelberg, Special classes of Boolean algebras, Handbook on Boolean algebras, vol. 1, part I, Chapter 6 (J. D. Monk, ed.), North-Holland, Amsterdam, 1989, pp. 239-284.

6. D. Mayer and R. S. Pierce, Boolean algebras with ordered basis, Pacific J. Math. 10 (1960), 925-942.

7. J. Roitman, Superatomic Boolean algebras, Handbook on Boolean algebras, vol. 3, Part II, Chapter 19 (J. D. Monk, ed.), North-Holland, Amsterdam, 1989, pp. 719-740.

8. J. Roseinstein, Linear ordering, Academic Press, NY, 1982.

9. M. Rubin and S. Shelah, On the cardinality of superatomic subalgebra of an interval algebra, 1988 (unpublished).

10. R. Sikorski, Boolean algebras, Ergeb. Math. Grenzgeb (3), vol. 25, Springer-Verlag, Berlin, 1964.

Defartment of Mathematics, University Ben-Gurion of the Negev, BeER-SheVA, ISRAel

Department of Mathematics, Campus Box 322, Université Aix-Marseille 3, F 13397 Marseille Cedex 13 France 